\title{
The Learning Styles Educational Neuromyth: Lack of Agreement Between Teachers' Judgments, Self-Assessment, and Students' Intelligence
}

\author{
Marietta Papadatou-Pastou ${ }^{1 *}$, Maria Gritzali $^{1}$ and Alexia Barrable ${ }^{2}$ \\ ${ }^{1}$ Department of Primary Education, School of Education, National and Kapodistrian University of Athens, Athens, Greece, \\ ${ }^{2}$ School of Education and Social Work, University of Dundee, Scotland, United Kingdom
}

OPEN ACCESS

Edited by:

Ana Lucia Pereira,

Universidade Estadual de Ponta

Grossa, Brazil

Reviewed by:

Roberta Ekuni,

State University of Northern Paraná,

Brazil

Katie Zhukov

The University of Queensland,

Australia

*Correspondence:

Marietta Papadatou-Pastou marietta.papadatou-pastou@ seh.oxon.org

Specialty section:

This article was submitted to Educational Psychology, a section of the journal

Frontiers in Education

Received: 26 September 2018 Accepted: 14 November 2018 Published: 29 November 2018

Citation:

Papadatou-Pastou M, Gritzali M and Barrable A (2018) The Learning Styles Educational Neuromyth: Lack of

Agreement Between Teachers Judgments, Self-Assessment, and Students' Intelligence. Front. Educ. 3:105.

doi: 10.3389/feduc.2018.00105
Learning styles (LS) have dominated educational practice since their popularization in the 1970s. Studies have shown that they are accepted by more than $90 \%$ of teachers worldwide. However, LS have also received extensive criticism from researchers and academics, due to the poor theoretical justification of the theory, their problematic measurement, and the lack of systematic studies supporting them. The present study tested the hypothesis that teachers' and students' assessment of preferred LS should correspond. Moreover, it tested whether teachers' judgment of LS is driven by the students' IQ. Both questions were studied for the first time in a systematic fashion within LS research in primary school pupils. Fifth and sixth grade pupils $(n=199)$ were asked to self-assess their preferred LS, while their teachers were asked to provide their own assessment on individual pupils' LS. No relationship was found between pupils' self-assessment and teachers' assessment, suggesting that teachers cannot assess the LS of their students accurately. Moreover, students' intelligence was not found to drive teachers' assessment of their LS. This study adds to the body of evidence that is skeptical of the adoption of LS in mainstream education.

Keywords: learning styles, auditory, visual, kinaesthetic, neuromyths, VAK, intelligence

\section{INTRODUCTION}

The term Learning Styles (LS) is used to describe the idea that different individuals differ in the modality of instruction that is most effective to them (Pashler et al., 2008). Criticism of the concept of LS has been widespread (Curry, 1990; Coffield et al., 2004; Geake, 2008) and in 2002 the Organization for Economic Cooperation and Development (OECD), through its Centre for Educational Research and Innovation (CERI), pronounced LS a neuromyth (OECD, 2002). The OECD classification was particularly concerned with the three LS that are often seen in educational practice, namely the visual, auditory, or haptic (kinaesthetic) types (OECD, 2002).

Despite the lack of evidence in support of the concept, LS remain ever popular with a great majority of educators. A study looking at teachers from the UK and the Netherlands showed that more than $90 \%$ of teachers believe there is an optimal delivery style for each learner (Dekker et al., 2012). Similar studies have found equally high numbers in Spain (Ferrero et al., 2016) and Portugal (Rato et al., 2013). In Greece, the setting for this current study, 97\% of practicing teachers believe 
that students' performance can be enhanced when material is delivered in an individual's preferred LS (Deligiannidi and Howard-Jones, 2015) and 94\% of student teachers agree (Papadatou-Pastou et al., 2017).

Only a few empirical studies have sought to shed light on the rather obscure picture (Marcus, 1977; Rogowsky et al., 2015). For example, Rogowsky et al. (2015) investigated the effect of LS preference in text comprehension in an adult sample. According to the findings, no statistical significance was to be found in the relationship between LS preference, mode of delivery, and learning aptitude.

Building upon this evidence, the current study was designed. Its main aim was to assess whether the LS of primary school aged pupils as assessed by the students and their teachers, would agree. These are important questions, as teachers typically adopt LS within a classroom context by relying on their own assessment of students LS (Cassidy, 2004; Graf and Liu, 2009). Moreover, there has been limited research done on primary-aged pupils (e.g., Sun et al., 2008), with research mainly available on older students or adult samples (Diaz and Cartnal, 1999; Massa and Mayer, 2006; Husmann and O'Loughlin, 2018). There is also very limited literature relating LS to IQ (Dunn and Price, 1980; Barbe and Milone, 1982; Griggs and Dunn, 1984; Dunn, 1990) and no studies investigating the hypothesis of whether teachers confuse their students' intellectual ability with a specific LS. However, there is previous research to suggest that teachers can erroneously associate IQ with other characteristics, such as being left-or righthanded (Papadatou-Pastou et al., 2017), or socio-economic status and gender (Auwarter and Aruguete, 2008).

In the current study, intellectual ability was measured by means of a fluid intelligence test, Raven's Colored Progressive Matrices (CPM; Raven et al., 2004), which is "the paradigm test of non-verbal, abstract reasoning ability" (Mackintosh, 1996, p. 564) and is widely regarded as one of the best tests of Spearman's $\mathrm{g}$, the general factor underlying all cognitive abilities (Spearman, 1946). Raven's matrices have been frequently used in educational research (Brouwers et al., 2009), and have been shown to have good construct validity across age, gender, and country (Rushton et al., 2003, 2004). Across cultures with a tradition of literacy, like the Greek culture, the norms for the RPM have been shown to be unexpectedly similar (Raven, 2008). Moreover, in contrast to fullscale IQ tests, such as the Wechsler Intelligence Scale for Children (WISC; Kaufman et al., 2015), it is easy to administer and can be completed through group administration.

To conclude, the main aim of the study was to investigate whether there is an association between primary school pupils' self-report of their preferred LS and teachers' evaluation of each pupil's LS. The second aim of the study was to investigate whether teachers' assessments of LS would be informed by their students' intellectual ability.

\section{METHODS}

\section{Participants}

One hundred and ninety nine 5th and 6th grade primary school students including 105 girls (mean age $=135.90$ months, SD $=7.27$, range $=125-149$ ) and 94 boys (mean age $=136.25$ months, $\mathrm{SD}=7.28$, range $=121-148$ ) participated in this study after their parents gave written informed consent. Five state schools in the first district of the municipality of Athens were recruited to take part in the study. Nineteen teachers (15 women; mean age $=50.52$ years, $\mathrm{SD}=5.24$, range $=31-55)$ also participated after giving written informed consent. Their mean teaching experience was 20.05 years $(\mathrm{SD}=4.05$, range $=7$ $25)$. Ten teachers taught 5 th grade and nine 6 th grade level. The study was granted ethical approval by the Institute of Educational Policy, supervised by the Greek Ministry of Education, Research, and Religious Affairs (protocol number: $\Phi 15 / 1181 / 174596 / \Delta 1$ ). Written informed consent was given in accordance with the Declaration of Helsinki.

\section{Instruments}

Forced-choice LS question. Student participants were asked whether they are auditory, visual, or kinaesthetic learners. Students had to circle among three choices "visual," "auditory," "kinaesthetic."

Raven's Colored Progressive Matrices (Raven et al., 2004). The $\mathrm{CPM}$ is a measure of fluid intelligent and is considered to be a culture-fair intelligence test (Van de Vijver and Hambleton, 1996). It comprises three sets of 12 items. For each item students have to identify a missing piece in a pattern, choosing among six possible options. Each set of items gets progressively harder. Raw scores were matched with each participant's chronological age in order to calculate IQ scores.

\section{Teachers Questionnaire}

Teachers were asked to respond to two items, namely "Does teaching that is tailor made to the students' LS reinforce the students' performance?", which was an open-ended question, and "Which is the learning style of each of your students?" with possible responses to the latter question being auditory, visual, or kinaesthetic. Each teacher provided only one LS for each student, after being prompted to recall specific incidents from the classroom. Each student's LS was judged by one teacher.

\section{Statistical Analysis}

All analyses were performed using the Statistical Package for the Social Sciences (SPSS) v.25. In order to analyze the qualitative data collected from the open-ended question, word cloud analysis was used, which graphically represents word frequency, giving greater prominence to words that appear more frequently in the participants' responses. In addition, the most characteristic segments of text were presented. The analysis was performed using Iramuteq (Ratinaud and Dejean, 2009), an R interface for multidimensional analysis of texts and questionnaires. Word clouds are increasingly being employed in exploratory qualitative analysis in order to identify the focus of written material (Atenstaedt, 2012). In order to investigate a possible association between the two types of LS assessment (student's self-assessment and teachers' assessment), $\chi^{2}$ analysis was performed. In order to test whether the three LS, as assessed by the teachers, differed in terms of IQ, analysis of variance (ANOVA) was performed, with the biological sex of the students and the 


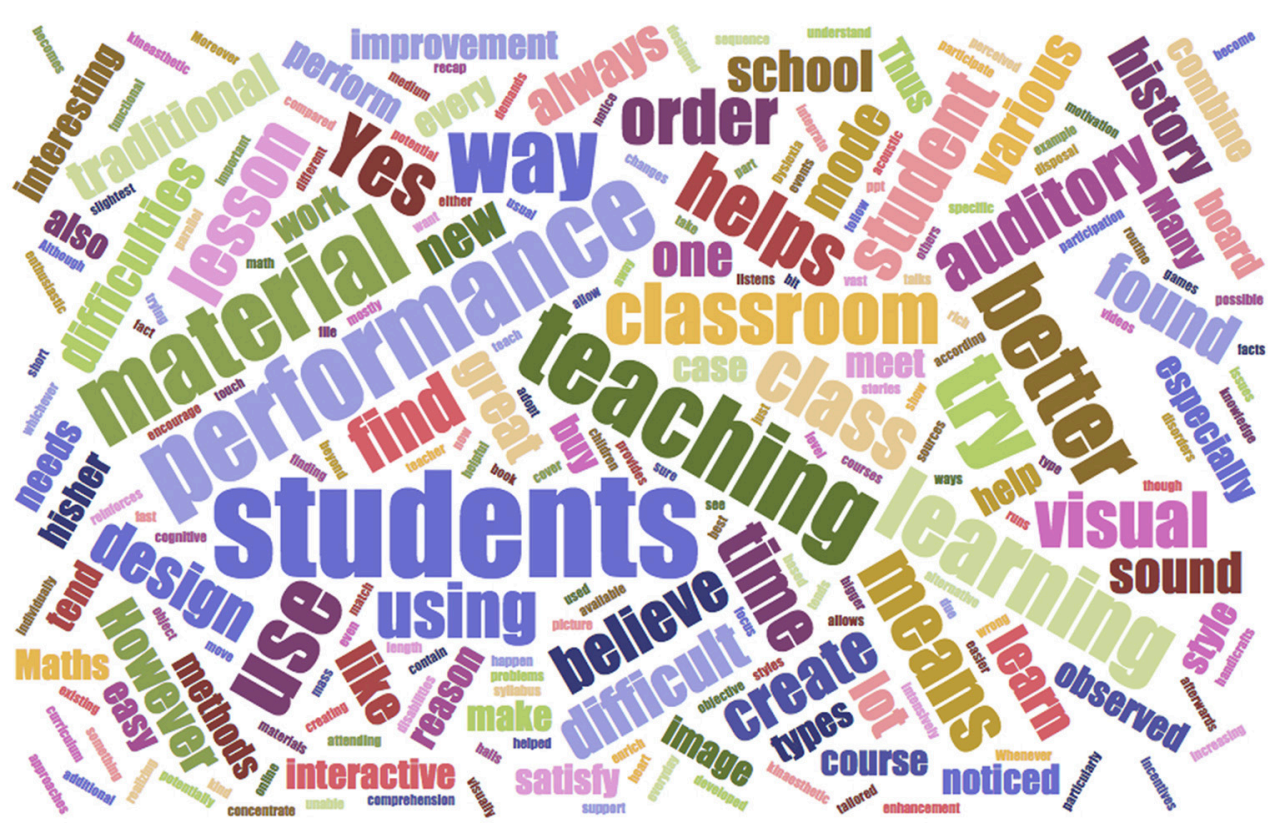

FIGURE 1 | Word cloud representing the most frequent words, giving greater prominence to words that appear more frequently, in the teachers' responses to the open-ended question: "Does teaching that is tailor made to the students' learning styles reinforce the students' performance?".

LS of the students according to the teachers as the grouping factors. Whether teachers adopted the LS styles myth could not be used, as all teachers reported that they believe in LS. The partial eta squared $\left(\eta^{2}\right)$ statistic was used as the effect size measure. All $p$-values were two-tailed and the $\alpha$-level was set at 0.05 . All data and quantitative analysis code are available in the Open Science Framework repository (https://osf. io/a9g7s/).

\section{RESULTS}

All teachers reported that they believed that teaching tailored to the students' LS enhances the intake of new information. However, only four teachers referred to the VAK explicitly, that is by using the words visual, auditory, and/or kinaesthetic. For example, one female teacher reported, "Yes, of course I try to support the students whom I have found out to be visual, auditory, or kinaesthetic types with material that I design myself or that I find online." Most teachers, however, referred to "learning styles" in a more general fashion or did not make it clear in their responses they referred to the VAK model. For example a male teacher reported "Students' performance is enhanced when using material that I create personally through handicrafts or through a computer." and a female teacher reported "Yes, teaching is tailored to the learning styles of the students sometimes and there is a great enhancement in their performance."

Figure 1 presents the word cloud stemming from the text of the teachers' responses to the open-ended question, after removing common words, such as "and." The words that were more prominent, as indicated by the size of the words in the word
TABLE 1 | Crosstabulation of Learning Styles (LS) as measured by self-assessment by the students and by the assessment of teachers.

\begin{tabular}{lcccc}
\hline \multicolumn{4}{c}{ Teachers' assessment } \\
\hline $\begin{array}{l}\text { Self-assessment } \\
\text { by students }\end{array}$ & Visual & Auditory & Kinaesthetic & Total \\
\hline Visual & 11 & 9 & 17 & 37 \\
Auditory & 7 & 15 & 17 & 39 \\
Kinaesthetic & 32 & 27 & 64 & 123 \\
Total & 50 & 51 & 98 & 199 \\
\hline
\end{tabular}

cloud were "students," "performance," "learning," “teaching," and "material."

Table 1 presents the LS of the students as assessed by self-assessment and by the teachers. A $\chi^{2}$ analysis was performed to test for their possible association. No statistically significant associations were found to exist; self-assessment-teacherassessment, $\chi_{(4)}^{2}=4.86, p=0.30$.

A $2 \times 3$ (ANOVA) was performed, with the biological sex of the students (male or female) and the LS of the students according to the teachers (visual, auditory, or kinaesthetic) as the grouping factors and the IQ score as the dependent variable. No main effect of LS was found, $F_{(2,198)}=0.38, p=0.69, \eta^{2}=0.004$ (mean raw Raven score for visual types $=30.86, \mathrm{SD}=3.60$, mean raw Raven score for auditory types $=30.27, \mathrm{SD}=4.45$, mean raw Raven score for kinaesthetic types $=30.16, \mathrm{SD}=4.73$ ) or main effect of sex, $F_{(1,198)}=0.21, p=0.65, \eta^{2}=0.001$ (mean raw Raven score for males $=30.29, \mathrm{SD}=4.51$, mean raw Raven score for females $=30.44, \mathrm{SD}=4.31$ ). No interaction was found between LS and sex, $F_{(2,198)}=1.20, p=0.30, \eta^{2}=0.012$. 


\section{DISCUSSION}

The present study looked at whether self-assessment and teacher assessment agreed in the identification of preferred LS in primary school-aged pupils. Results show that there is no correlation between the two. Findings, moreover, suggest that the teachers do not see intellectual ability as a proxy for a particular learning style. This was the first study to investigate these questions and one of the few studies within the LS literature to employ a sample of primary school students. It adds to the growing body of critical literature about the use of LS in educational settings (Coffield et al., 2004; Franklin, 2006; Pashler et al., 2008).

The present study focused at a certain type of LS, VAK, as it is very commonly used in primary schools, with each student's LS assessed by one teacher (Sharp et al., 2008). Teachers were asked in an open-ended question whether they believed that teaching tailored to the students' LS enhances the intake of new information. The phrasing of this question did not refer specifically to the VAK typology, and this was also reflected in the teachers' responses, as only four teachers referred to the VAK model per se. The rest of the teachers referred to "learning types" in a more general manner, possibly reflecting the vague nature of the concept. This was a limitation of the present study, as we could not ascertain if the teachers adopted the VAK model specifically and could further not test for possible differences between those teachers who adopt the model and those who do not. Future studies should add a question on the VAK typology, as it could be the case that teachers believe in LS, but not

\section{REFERENCES}

Atenstaedt, R. (2012). Word cloud analysis of the BJGP. Br. J. Gen. Pract. 62:148. doi: 10.3399/bjgp12X630142

Auwarter, A. E., and Aruguete, M. S. (2008). Effects of student gender and socioeconomic status on teacher perceptions. J. Educ. Res. 101, 242-246. doi: 10.3200/JOER.101.4.243-246

Barbe, W. B., and Milone, M. N. Jr. (1982). Modality characteristics of gifted children. Gifted Child Today 5, 2-5.

Brouwers, S. A., Van de Vijver, F. J., and Van Hemert, D. A. (2009). Variation in Raven's progressive matrices scores across time and place. Learn. Individ. Differ. 19, 330-338. doi: 10.1016/j.lindif.2008.10.006

Cassidy, S. (2004). Learning styles: an overview of theories, models, and measures. Educ. Psychol. 24, 419-444. doi: 10.1080/0144341042000228834

Coffield, F., Moseley, D., Hall, E., and Ecclestone, K. (2004). Learning Styles and Pedagogy in Post-16 Learning: A Systematic and Critical Review. London: Learning and Skills Research Centre.

Curry, L. (1990). A critique of the research on learning styles. Educ. Leader. 48, 50-56.

Dekker, S., Lee, N. C., Howard-Jones, P., and Jolles, J. (2012). Neuromyths in education: prevalence and predictors of misconceptions among teachers. Front. Psychol. 3:429. doi: 10.3389/fpsyg.2012.00429

Deligiannidi, K., and Howard-Jones, P. (2015). The neuroscience literacy of teachers in Greece. Proc. Soc. Behav. Sci. 174, 3909-3915. doi: 10.1016/j.sbspro.2015.01.1133

Diaz, D. P., and Cartnal, R. B. (1999). Students' learning styles in two classes: online distance learning and equivalent on-campus. Coll. Teach. 47, 130-135. doi: 10.1080/87567559909595802

Dunn, R. (1990). Understanding the Dunn and Dunn learning styles model and the need for individual diagnosis and prescription. Read. Writ. Q. 6, 223-247. doi: 10.1080/0748763900060303 specifically to the VAK model. Moreover, judgments made by different teachers for the same students could be compared.

Overall, we posit that identifying preferred LS can be a hitand-miss process, with no agreement between the assessment made by teachers and students. We suggest that if the identification of LS, as they are currently understood and used within primary education, is unreliable, as evident by the findings of the present study, this should constitute an additional reason why teachers should abandon the use of LS in instruction. Our study thus adds to the growing body of literature against the use of LS in education. Moreover, debunking the myth of LS as well as educating teachers in the use of evidence-based practices is recommended.

\section{ETHICS STATEMENT}

All subjects gave written informed consent in accordance with the Declaration of Helsinki. The study was granted ethical approval by the Institute of Educational Policy, supervised by the Greek Ministry of Education, Research and Religious Affairs.

\section{AUTHOR CONTRIBUTIONS}

MP-P conceived and designed the study. $\mathrm{AB}$ was consulted at the initial stages. MG collected the data. MP-P analyzed the data. $\mathrm{AB}$ and MP-P did the drafting, and revising of the work, and wrote the final manuscript. MP-P supervised the project from conception to submission.

Dunn, R. S., and Price, G. E. (1980). The learning style characteristics of gifted students. Gifted Child Q. 24, 33-36. doi: 10.1177/001698628002400107

Ferrero, M., Garaizar, P., and Vadillo, M. A. (2016). Neuromyths in education: prevalence among Spanish teachers and an exploration of cross-cultural variation. Front. Hum. Neurosci. 10:496. doi: 10.3389/fnhum.2016.00496

Franklin, S. (2006). VAKing out learning style why the notion of 'learning styles' is unhelpful to teachers. Education 34, 81-87. doi: 10.1080/03004270500507644

Geake, J. (2008). Neuromythologies in education. Educ. Res. 50, 123-133. doi: 10.1080/00131880802082518

Graf, S., and Liu, T.-C. (2009). Supporting teachers in identifying students' learning styles in learning management systems: an automatic student modelling approach. J. Educ. Technol. Soc. 12, 3-14.

Griggs, S. A., and Dunn, R. S. (1984). Selected case studies of the learning style preferences of gifted students. Gifted Child Q. 28, 115-119. doi: $10.1177 / 001698628402800304$

Husmann, P. R., and O'Loughlin, V. D. (2018). Another nail in the coffin for learning styles? Disparities among undergraduate anatomy students' study strategies, class performance, and reported VARK learning styles. Anat. Sci. Educ. doi: 10.1002/ase.1777. [Epub ahead of print].

Kaufman, A. S., Raiford, S. E., and Coalson, D. L. (2015). Intelligent Testing With the WISC-V. John Wiley \& Sons.

Mackintosh, N. J. (1996). Sex differences and IQ. J. Biosoc. Sci. 28, 559-572. doi: $10.1017 /$ S0021932000022586

Marcus, L. (1977). How teachers view student learning styles. NASSP Bull. 61, 112-114. doi: 10.1177/019263657706140821

Massa, L. J., and Mayer, R. E. (2006). Testing the ATI hypothesis: should multimedia instruction accommodate verbalizer-visualizer cognitive style? Learn. Individ. Differ. 16, 321-335. doi: 10.1016/j.lindif.2006.10.001

OECD (2002). Organisation for Economic Co-operation and Development. Understanding The Brain: Towards a New Learning Science. Paris: OECD Publishing. 
Papadatou-Pastou, M., Haliou, E., and Vlachos, F. (2017). Brain knowledge and the prevalence of neuromyths among prospective teachers in Greece. Front. Psychol. 8:804. doi: 10.3389/fpsyg.2017.00804

Pashler, H., McDaniel, M., Rohrer, D., and Bjork, R. (2008). Learning styles: concepts and evidence. Psychol. Sci. Public Interest 9, 105-119. doi: 10.1111/Fj.1539-6053.2009.01038.x

Ratinaud, P., and Dejean, S. (2009). IRaMuTeQ: Implémentation de la Méthode ALCESTE d'Analyse de Texte Dans un Logiciel Libre. Toulouse: Modélisation Appliquée aux Sciences Humaines et Sociales (MASHS2009)

Rato, J. R., Abreu, A. M., and Castro-Caldas, A. (2013). Neuromyths in education: what is fact and what is fiction for Portuguese teachers? Educ. Res. 55, 441-453. doi: 10.1080/00131881.2013.844947

Raven, J. (2008). “The Raven progressive matrices tests: their theoretical basis and measurement model," in Uses and Abuses of Intelligence. Studies Advancing Spearman and Raven's Quest for Non-arbitrary Metrics (Raven), 17-68.

Raven, J., Raven, J. C., and Court, J. H. (2004). Manual for Raven's Progressive Matrices and Vocabulary Scales. Section 3: Standard Progressives Matrices: 1998 Edition. San Antonio, TX: Harcourt Assessment.

Rogowsky, B. A., Calhoun, B. M., and Tallal, P. (2015). Matching learning style to instructional method: effects on comprehension. J. Educ. Psychol. 107, 64-78. doi: $10.1037 / \mathrm{a} 0037478$

Rushton, J. P., Skuy, M., and Bons, T. A. (2004). Construct validity of Raven's advanced progressive matrices for African and non-African engineering students in South Africa. Int. J. Select. Assess. 12, 220-229. doi: 10.1111/j.0965-075X.2004.00276.x
Rushton, J. P., Skuy, M., and Fridjhon, P. (2003). Performance on Raven's advanced progressive matrices by African, East Indian, and white engineering students in South Africa. Intelligence 31, 123-137. doi: 10.1016/S0160-2896(02) 00140-X

Sharp, J. G., Byrne, J., and Bowker, R. (2008). The trouble with VAK. Educ. Fut. 1, 89-97.

Spearman, C. (1946). Theory of general factor. Br. J. Psychol. 36, 117-131.

Sun, K. T., Lin, Y. C., and Yu, C. J. (2008). A study on learning effect among different learning styles in a web-based lab of science for elementary school students. Comput. Educ. 50, 1411-1422. doi: 10.1016/j.compedu.2007. 01.003

Van de Vijver, F., and Hambleton, R. K. (1996). Translating tests. Eur. Psychol. 1, 89-99. doi: 10.1027/1016-9040.1.2.89

Conflict of Interest Statement: The authors declare that the research was conducted in the absence of any commercial or financial relationships that could be construed as a potential conflict of interest.

Copyright (C) 2018 Papadatou-Pastou, Gritzali and Barrable. This is an open-access article distributed under the terms of the Creative Commons Attribution License (CC $B Y)$. The use, distribution or reproduction in other forums is permitted, provided the original author(s) and the copyright owner(s) are credited and that the original publication in this journal is cited, in accordance with accepted academic practice. No use, distribution or reproduction is permitted which does not comply with these terms. 